\title{
MMW-HOTLINE
}

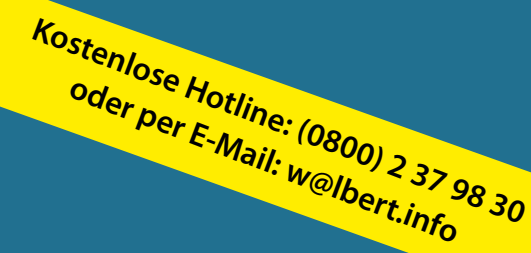

Leser der MMW können sich mit allen Fragen zur Abrechnung und Praxisführung an Helmut Walbert, Facharzt für Allgemeinmedizin, Würzburg, wenden. Sie erreichen inn jeden Donnerstag von 13 bis 15 Uhr unter der kostenlosen Rufnummer (0800) 2379830 oder per E-Mail: w@lbert.info.

Helmut Walbert

Allgemeinarzt, Medizinjournalist und Betriebswirt Medizin

\section{Wie schütze ich mich bei Ersatzverordnungen vor dem Regress?}

Dr. med. K. H., Allgemeinarzt, KV Hamburg: Ich muss derzeit wegen des Rückrufes der Adrenalin-Injektoren Anapen ${ }^{\circledR}$ und Anapen junior ${ }^{\circledR}$ Ersatzverordnungen zulasten meines Arzneimittelbudgets vornehmen. Wie kann ich mich vor eventuellen Regressen schützen?

Antwort: Laut "Rote Hand Brief", besteht ein mögliches Risiko, dass bei der Anwendung der Injektoren keine Adrenalinlösung abgegeben wird. Als verordnender Arzt tragen Sie eine Mitverantwortung für Ihre Patienten. Sie sind nicht nur zur Ersatzverordnung verpflichtet, sondern haben sogar eine Informationspflicht gegenüber Ihren Patienten.

Der Hersteller ist offensichtlich nicht bereit, die Kosten für die Ersatzbeschaffung zu übernehmen. Aus diesem Grund empfehle ich auf dem Rezept den Zusatz „Ersatz/Rückruf" anzubringen. Damit sind diese Rezepte im Falle einer Richtgrößenprüfung auf jeden
Fall gekennzeichnet. Im Prinzip wäre es Angelegenheit der „zahlenden“ Krankenkassen, die entsprechende Firma für die Mehrkosten „in Regress" zu nehmen. Auch der Eigenanteil für den Patienten müsste eigentlich über die Krankenkasse geregelt werden. Dies ist aber Sache unserer "mündigen" Patienten. Wir sollten uns auf die Sicherstellung der Wirksamkeit im Notfall beschränken.

\section{Sprechstundenbedarf}

\section{Läuft die Abrechnung jetzt über eine zentrale Abrechnungsstelle?}

\begin{abstract}
Dr. med. P. G., Allgemeinarzt, KV WestfalenLippe:

Ich habe gehört, dass die Abrechnung des Sprechstundenbedarfs nicht mehr über die AOK, sondern über eine zentrale Abrechnungsstelle läuft. Ist das richtig? Was steckt dahinter?

Antwort: In Ihrem Fall ist das richtig. Es gibt eine zentrale Rezeptprüfungsstelle für die Abrechnung des Sprechstundenbedarfs in Duderstadt. Einzig der Freistaat Bayern macht auch hier eine Ausnahme!

Der Hintergrund ist nicht nur Zentralisierung und damit möglicherweise Kosteneinsparung im Verwaltungsbereich. Es geht auch um Kosteneinsparung bei den Verord-
\end{abstract}

nungen. Vor allem geht es um die Überprüfung der Zulässigkeit der über den Sprechstundenbedarf ausgestellten Verordnungen und um die Plausibilität der Verordnung in der Menge zur Praxisgröße und Praxisstruktur.

Da die Vereinbarungen zum Sprechstundenbedarf auf Landesebene erfolgen, ist es notwendig, von Zeit zu Zeit die Praxisliste auf Übereinstimmung mit der aktuellen KV-Liste zu überprüfen.

Es ist wichtig zu beachten, dass über Sprechstundenbedarf Medikamente (z. B. Ampullen) nur für die Notfallbehandlung bestellt werden dürfen. Erhalten Patienten bestimmte Mittel wie beispielsweise B12-Injek- tionen bei Perniciosa regelmäßig gespritzt, dann müssen diese Medikamente auf den Namen des Patienten zulasten der betreffenden Krankenkasse individuell (nach-) verordnet werden. Notfallmedikamente dienen der unmittelbaren Versorgung von einzelnen Notfällen. Großpackungen von Ampullen von Schmerzmitteln mögen zwar pro Ampulle wesentlich billiger sein, sind aber regressgefährdet, weil sie den entsprechenden Patienten und ihren Krankenkassen nicht zuordnungsfähig sind. Darüber hinaus entfällt die Selbstbeteiligung der Patienten, die in der Regel die Richtgrößen der Praxis entlastet. 